\title{
Surgical Management of 100 Primary Carcinomas of the Liver: Prognostic factors after hepatic resection for primary liver cancer
}

\author{
Manabu Yamamura*, Shoji Uetsuji*, Yoshinori Okuda, \\ Keigo Yamamichi, Yoshifumi Kise, Hisanao Komada, \\ Masanori Kwon, Yoshiaki Inada, Toshiyuki Minoura, \\ Takeo Yamada and Masakatsu Yamamoto* \\ Department of Surgery and Institute for Liver Research*, \\ Kansai Medical University \\ Moriguchi, Osaka 570, Japan
}

(Received for publication August 31, 1990)

Key words : Primary liver cancer, Surgical management, Prognostic factors

\begin{abstract}
Between January 1980 and December 1989, surgical treatment of primary liver cancer was performed in 100 cases including two reoperations. Of these 89 were male and 11 female; their ages ranged from 5 months to 74 years. Pathological findings showed that 92 of the patients had hepatocellular carcinoma, 6 cholangiocellular carcinoma, and 2 hepatoblastoma; in $85 \%$ of the cases these were associated with cirrhosis. Of the 100 patients, 52 underwent typical resection of the liver, 28 limited resection, and 20 no resection. Follow-up results in resected cases showed that the cumulative survival rates at 1,3 , and 5 years were $66.6 \%$, $46.7 \%$, and $36.7 \%$, respectively. Non-resected patients died within 3 years. Various factors were evaluated by analysis in respect to cumulative survival, after exclusion of operative deaths, to clarify prognosis after hepatic resection. Factors affecting the outcome were gross tumor findings including tumor size. A better prognosis was associated with encapsulated nodular forms, a tumor size of less than $2 \mathrm{~cm}$, and no portal vein and hepatic serosal involvement in the resected specimen.
\end{abstract}

\section{Introduction}

Malignant neoplasms of the liver, primary or metastatic, are generally lethal. Recent diagnostic advances including application of tumor markers such as alpha-fetoprotein (AFP) and carcinoembryonic antigen (CEA), and diagnostic imaging such as ultrasonography (US), 
and computerized tomography (CT) have, however, increased the chance of detecting early hepatic tumors. With malignant lesions, the therapeutic value of hepatic resection has been proven in the clinical field ${ }^{5}, 35$. Despite this fact such operations are still confined to only a few major medical centers and operative risk remains higher than with other abdominal surgical procedures.

In Japan, the major indication for hepatic resection is hepatocellular carcinoma (HCC). However, the incidence of concomitant cirrhosis is much higher than in Western Countries 33, 38-40) and in resecting cirrhotic livers the surgeon encounters several major problems ${ }^{21)}$. Poor functional reserve and technical difficulty are of particular interest to the improvement of operative results. The major causes of death in long-term follow-up of HCC patients after hepatectomy were found to be almost equally tumor recurrence, with growth in local and/or distant sites or hepatic failure and rupture of esophageal varices due to severe liver cirrhosis and involvement of the portal trunk and portal hypertension.

Improvement in the assessment of $\mathrm{HCC}$ resectability ${ }^{25,26,30,34)}$ have led to a reduction in operative mortality rates. Thus the remarkable decrease is generally attributed to appropriate evaluation of hepatic functional reserve, better pre- and post-operative management, and new surgical techniques such as ultrasonically guided hepatectomy ${ }^{24)}$, and others ${ }^{7,17)}$ as well as early diagnosis. In a previous report from The Liver Cancer Study Group of Japan ${ }^{33)}$, where the operative death rate was $15.7 \%$, approximately $60 \%$ of the operative procedures were extended and conventional lobectomy. Limited resections, which have become widely performed according to a recent report ${ }^{40)}$, mainly because of the developments mentioned above, have greatly contributed to lowering of the operative mortality.

In the last 10 years up to the end of 1989, we have treated 100 patients with primary liver cancer by various surgical procedures. In this report, the long-term follow-up results of 80 patients surviving after hepatic resection and 20 patients without hepatic resection have been reviewed with a retrospective analysis of some of the factors that influenced prognosis.

\section{Materials and Methods}

\section{Subjects}

Over a 10 year period (January 1980 to December 1989), a total of 100 patients diagnosed as having primary liver cancer underwent surgery in the Surgical Department of Kansai Medical University Hopital. They included 89 men and 11 women. Of the patients 98 were adults ranging in age from 32 to 74 years (mean age, 55.8 years), and two were children aged 5 months and 2 years, respectively. 
The preliminary diagnoses were established clinically by abnormal biochemical data with elevated tumor marker levels, US, CT, magnetic resonance imaging (MRI), and hepatic angiography.

Decisions on surgery and the extent of hepatic resection were based on estimation of size and anatomic location of tumor(s) and also the hepatic function. Patients were excluded if they had evidence of extrahepatic distant metastasis, more than two tumors in the bilateral hepatic lobes, a tumor thrombus in the main portal vein detected by US, CT, MRI, and angiography, or evidence of hepatic decompensation such as uncontrolled ascites, jaundice caused by hepatocellular damage or a history of hepatic encephalopathy.

\section{Measurement of serum AFP, CEA and HBsAg}

Serum AFP, CEA and HBsAg were measured by turbimetric immunoassay (TIA), enzyme immunoassay (EIA) and radioimmunoassay (RIA) using commercial kits.

\section{Histopathological diagnosis}

Histologic diagnoses were made on resected specimens and biopsy material obtained at laparotomy. The microscopic classification of primary liver cancers was based on apparent origin from hepatic parenchymal cells, biliary cells or others. Cirrhosis of the liver was diagnosed macroscopically at surgery and confirmed by histologic examination of non-tumorous parts of the liver adjacent to the tumor.

\section{Surgical procedures}

Laparotomy was performed on all primary liver cancer cases, including these undergoing hepatic resection, hepatic artery ligation, catheterization of the hepatic artery for chemotherapy, or for exploratory purposes. Typical hepatic resections were defined as trisegmentectomy, extended lobectomy, lobectomy, central bisegmentectomy and segmentectomy. Limited hepatic resection was defined as any kind of nonsegmental resection including subsegmentectomy and partial resection such as wedge-shape resection, spindle-shape resection, cone-shape resection, and enucleation. Operative mortality after hepatic resection was defined as death within 30 days of surgery.

\section{Patient survival}

All patients were followed until death or the end of June, 1990, at least six months after the last surgery. No patients were lost from the follow-up. Survival time was expressed in days starting from the day of operation and terminating on either the day of death or June 30, 1990, in cases of survivors.

\section{Factors possibly influencing patient survival}

Excluding 8 operative deaths, there were 72 cases available for analysis. All reasonable possible factors that might influence patient survival were investigated:gross morphologic 
appearance of tumor according to Eggel's classification ${ }^{23)}$, gross findings on tumor appearance, tumor size, status of accompanying liver cirrhosis, surgical procedures, and normal tissue margin taken with tumor specimen.

In evaluating gross findings of tumor appearance, a round shape with capsule formation was considered typical. Otherwise, an irregular tumor outline, satellite nodule formation surrounding the main tumor, tumor invasion to a branch of the portal or hepatic vein, tumor exposure at the surface of the liver, tumor invading surroundind organs or dissemination to the peritoneum, positive lymph node metastasis, or any combination to the above findings were assumed to be atypical.

Tumor size was expressed in centimeters as the longest diameter of the largest cut surface of the tumor and then classified into three subgroups : tumor size less than $2 \mathrm{~cm}$, from $2.1 \mathrm{~cm}$ to $5.0 \mathrm{~cm}$, above $5.1 \mathrm{~cm}$.

Adequate margin of resected specimens was defined as the shortest distance from the tumor to the resected section line and classified into two groups: one with an adequate margin exceeding or equal to $1 \mathrm{~cm}$ and the other termed inadequate of less than $1 \mathrm{~cm}^{22)}$.

\section{Statistical analysis}

Survival was analyzed by the Kaplan-Meier method ${ }^{12)}$ and survival curves were compared by the generalized Wilcoxon test ${ }^{8)}$. A p value less than 0.05 was considered statistically significant.

\section{Results}

\section{Histological type of primary liver cancer}

92 of 100 cases of primary liver cancer were revealed to be of hepatocellular carcinoma type. Of the remaining eight cases, six were cholangiocellular carcinomas (CCC) and two were hepatoblastomas.

\section{Age and sex distribution}

Table 1 and 2 show histological type of primary liver cancer with regard to sex or age distribution of the patients. Only 11 cases with primary liver cancer were female and male/female ratio was 7.4 to 1 . The peak incidence was in the range 50-59 years old for HCC and 60-69 years old for CCC. Mean ages were 56. $4 \pm 9.4$ years for HCC, 58. $8 \pm 10.2$ years for CCC (mean $\pm \mathrm{SD})$. Hepatoblastoma occurred prior to 3 years of age and the mean age was 17 months.

\section{Liver cirrhosis, serum HBsAg, and serum AFP and CEA}

Liver cirrhosis was present in $78 \%$ of cases macroscopically and in 85 of the 100 primary liver cancer patients microscopically. Serum HBsAg was positive in $23 \%$. No cirrhosis was 
Table 1 Histological Diagnosis and Numbers of Cases by Sex

\begin{tabular}{lccc}
\hline Histological Diagnosis & Male & Female & Total \\
\hline Hepatocellular Ca. & 81 & 11 & 92 \\
Cholangiocellular Ca. & 6 & 0 & 6 \\
Hepatoblastoma & 2 & 0 & 2 \\
\hline Total & 89 & 11 & 100 \\
\hline
\end{tabular}

$\mathrm{Ca} .=$ Carcinoma

Table 2 Type of Tumor and Age Distribution

\begin{tabular}{ccccc}
\hline $\begin{array}{l}\text { Range } \\
\text { (Years) }\end{array}$ & \multicolumn{2}{c}{ Histologic classification } & \multirow{2}{*}{ Total } \\
\cline { 2 - 4 } $0-9$ & HCC & CCC & HB & \\
\hline $10-19$ & 0 & 0 & 2 & 2 \\
$20-29$ & 0 & 0 & 0 & 0 \\
$30-39$ & 0 & 0 & 0 & 0 \\
$40-49$ & 5 & 0 & 0 & 5 \\
$50-59$ & 15 & 2 & 0 & 17 \\
$60-69$ & 34 & 0 & 0 & 34 \\
$70-79$ & 32 & 3 & 0 & 35 \\
\hline Total & 6 & 1 & 0 & 7 \\
\hline
\end{tabular}

$\mathrm{HCC}=$ Hepatocellular carcinoma,$\quad \mathrm{CCC}=$ Cholangiocellular carcinoma

$\mathrm{HB}=$ Hepatoblastoma

observed only in $11(12.0 \%)$ patients with HCC, $3(50.0 \%)$ patients with CCC and in both of the hepatoblastoma cases. The serum AFP was less than $10 \mathrm{ng} / \mathrm{ml}$ in 12 cases (13.0\%) and over $100 \mathrm{ng} / \mathrm{ml}$ in 44 cases $(47.8 \%)$ of the $92 \mathrm{HCC}$ patients. Serum CEA elevated above $10 \mathrm{ng} / \mathrm{mlwas}$ observed in $2(33.3 \%)$ patients with $\mathrm{CCC}$ and $3 .(3.3 \%)$ patients with HCC.

\section{Surgical procedures}

Of the 100 laparotomized patients (see Table 3), 80 underwent surgical resection of tumor, the remaining 20 having hepatic artery ligation (4 cases), arterial catheterization for chemotherapy (6 cases), and exploratory laparotomy only (10 cases). Of the 80 hepatic resections, 4 were trisegmentectomies or extended lobectomies, 23 were lobectomies or central bisegmentectomies, 25 were segmentectomies, 13 were subsegmentectomies, and 15 were 
Table 3 Surgery Indicated for Treatment of Primary Liver Cancer

\begin{tabular}{lcccc}
\hline \multicolumn{1}{c}{ Indication } & HCC & CCC & HB & Total \\
\hline Hepatic resection & 77 & 1 & 2 & 80 \\
Arterial ligation & 3 & 1 & 0 & 4 \\
Arterial catheterization & 4 & 2 & 0 & 6 \\
Exploratory laparotomy & 8 & 2 & 0 & 10 \\
\hline \multicolumn{1}{c}{ Total } & 92 & 6 & 2 & 100 \\
\hline
\end{tabular}

$\mathrm{HCC}=$ Hepatocellular carcinoma,$\quad \mathrm{CCC}=$ Cholangiocellular carcinoma

$\mathrm{HB}=$ Hepatoblastoma

Table 4 Operative Procedures Performed for Resection of Primary Liver Cancer.

\begin{tabular}{ccccc}
\hline Type of resection & No. of cases & HCC & CCC & HB \\
\hline $\begin{array}{c}\text { Typical resection } \\
\text { Trisegmentectomy and } \\
\quad \text { Extended lobectomy }\end{array}$ & 52 & $3(1)$ & 0 & 1 \\
$\begin{array}{c}\text { Lobectomy and } \\
\text { Bisegmentectomy }\end{array}$ & $21(5)$ & 1 & 1 \\
$\quad$ Segmentectomy & 25 & 0 & 0 \\
Limited resection & 28 & 13 & 0 & 0 \\
$\quad$ Subsegmentectomy & $15(2)$ & 0 & 0 \\
$\quad$ Partial resection & 80 & $77(8)$ & 1 & 2 \\
\hline Total & & & & \\
\hline
\end{tabular}

Figures in parentheses indicate the numbers of operative deaths.

$\mathrm{HCC}=$ Hepatocellular carcinoma, $\mathrm{CCC}=$ Cholangiocellular carcinoma

$\mathrm{HB}=$ Hepatoblastoma

partial resection (Table 4). Thus, typical resection was performed in 52 (65\%) cases and limited resection in $28(35 \%)$ cases. The operative mortality rate defined as the deaths within 30 days after hepatic resection was $10 \%$. Cause of death were disseminated intravascular coagulation resulted with massive bleeding during the operation (one case), acute renal failure with uncontrolled lymphorrhea (one case), ruptured esophageal varices (one case), hepatic failure (three cases), and multiple organ failure with intraperitoneal infection (two cases). 


\section{Patient survival}

The cumulative survival rates for the 80 patients undergoing hepatic resection were $59.5 \%$ at 500 days, $46.9 \%$ at 1000 days, $38.7 \%$ at 1500 days, and $36.6 \%$ at 2000 days. However, in the 20 cases for which resection was not performed, 500 day survival rate was $20.0 \%$ and all patients without hepatic resection died within 1000 days after laparotomy (Fig. 1). The survival rates between resection and nonresection cases were

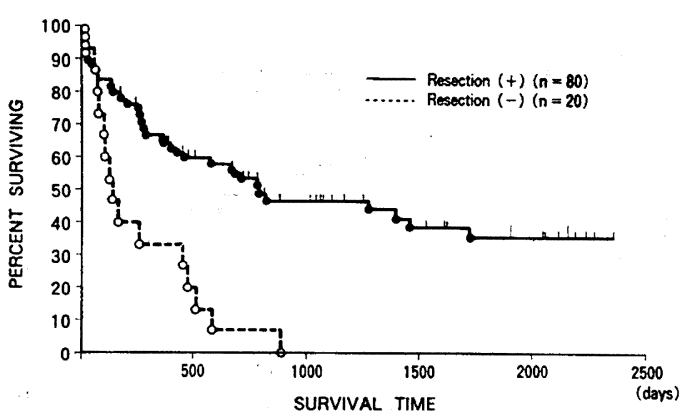

Fig. 1 Cumulative survival rates for laparotomized primary liver cancer patients with or without tumor resection. significantly different $(P<0.01)$.

\section{Factors that affected the patient's survival}

Excluding 8 operative deaths, a long-term follow-up study after hepatic resection of possible factors related to survival was possible in 72 cases. Following Eggel's classification of the macroscopic distribution of primary liver cancers, as shown in Fig. 2, 60 were considered to be "nodular", 12 were "massive", and none were "diffuse". The prognosis of the nodular type of tumor with expansive growth was better than that of the massive type with an irregular tumor outline $(P<0.01)$. Encapsulation of tumors has also associated with better survival $(P<0.05)$, i. e. typical gross finding tumors had a better prognosis as illustrated in Fig. 2 and 3. Tumor invasion to the branch of the portal vein (Fig. 4), exposure at the liver surface and invasion of extrahepatic surrounding tissues (Fig. 5) were noted to be associated with poorer survival rates $(\mathrm{P}<0.01$ and $\mathrm{P}<0.05$, respectively). However, satellite nodule formation in tissue surrounding the main tumor and tumor invasion into the capsule did not affect patient outcome (Figs. 6 and 7).

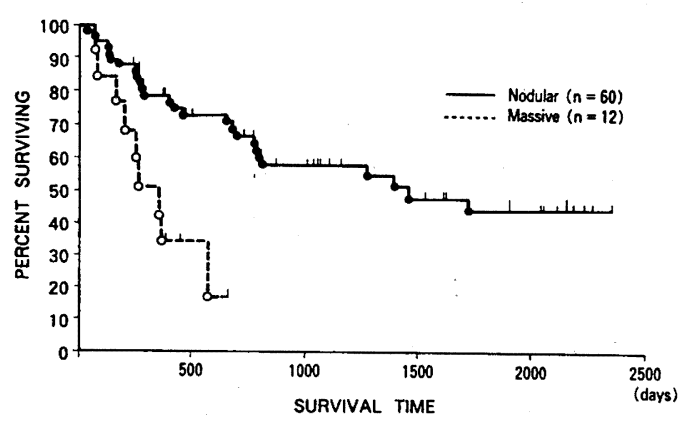

Fig. 2 Cumulative survival rates of resected cases according to Eggel's classificatin.

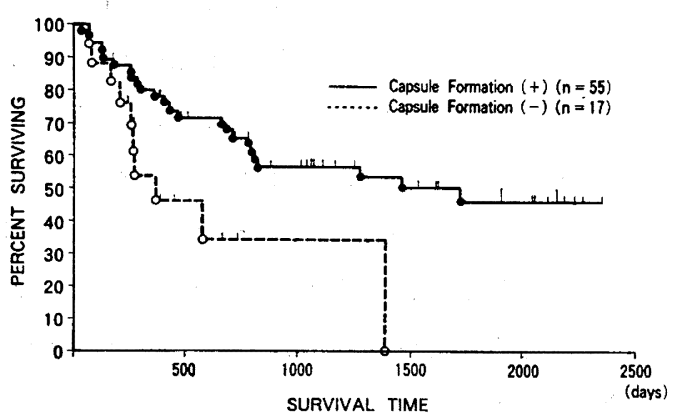

Fig. 3 Cumulative survival rates of resected cases according to capsule formation. 


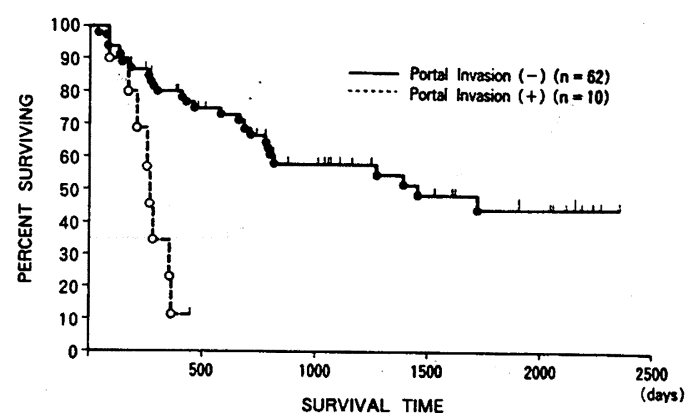

Eig. 4 Cumulative survival rates of resected cases according to portal invasion.

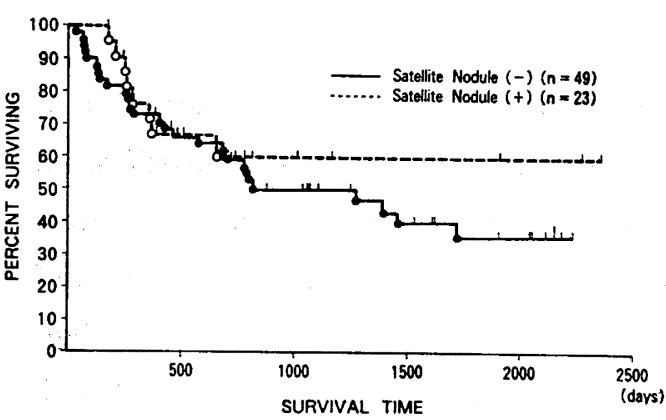

Fig. 6 Cumulative survival rates of resected cases according to satelite nodule formation.

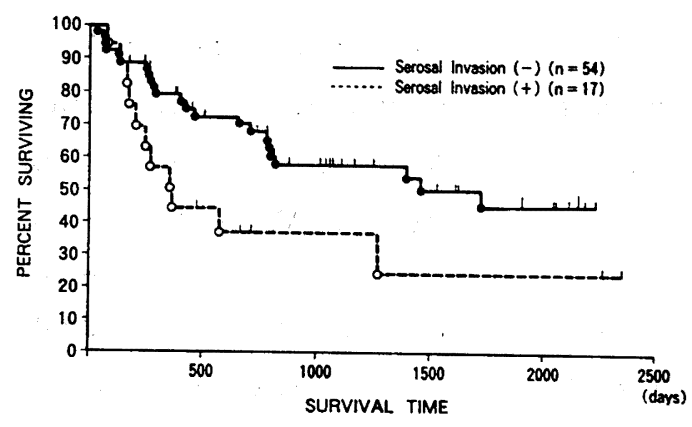

Fig. 5 Cumulativo survival rates of resected cases according to extrahepatic invasion.

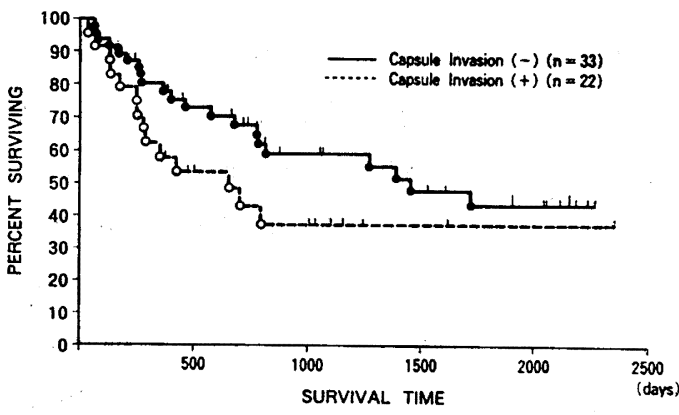

Fig. 7 Cumulative survival rates of resected cases according to capsular invasion.

In the 72 cases undergoing resection, survival rates were generally correlated with tumor size : tumor diameters of less than $2 \mathrm{~cm}$, were associated with a better prognosis than those over $5.1 \mathrm{~cm}(\mathrm{P}<0.01)$. The 4-year survival rates were significantly different by the KaplanMeier method $(P<0.05): 71.7 \%$ in the $<2.0 \mathrm{~cm}$ group, $37.8 \%$ in the 2.1 to $5.0 \mathrm{~cm}$ group, and $32.2 \%$ in the over $5.1 \mathrm{~cm}$ group. (Fig. 8)

The preoperative serum AFP level did not appear to influence the prognosis of primary liver cancer patients. The survival rate of patients with AFP below $100 \mathrm{ng} / \mathrm{ml}$ was slightly higher than those with over $100 \mathrm{ng} / \mathrm{ml}$. (Fig. 9) Coexistent hepatic cirrhosis did not significantly affect survival rates in primary liver cancer patients (Fig. 10) and also no significant overall difference was observed regarding preoperative indocyanine green (ICG) clearances of above or below $20 \%$ at $15 \mathrm{~min}$. (Fig. 11). However, patients with ICG 15 values below $20 \%$ showed better long-term survival rates as compared with their counterparts with values above $20 \%$ by the Kaplan-Meier method $(P<0.01)$. No significant overall differences were 


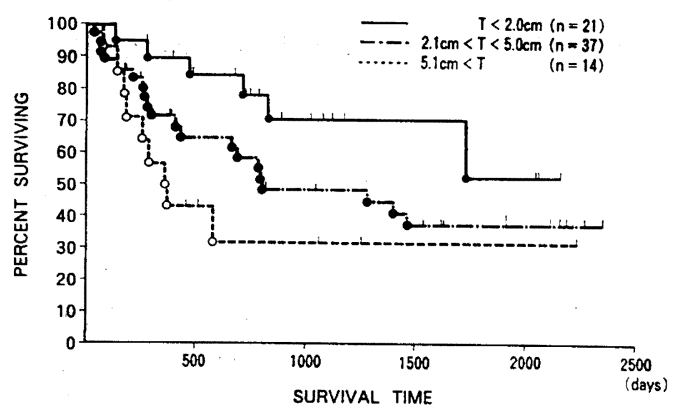

Fig. 8 Cumulative survival rates of resected cases according to tumor size.

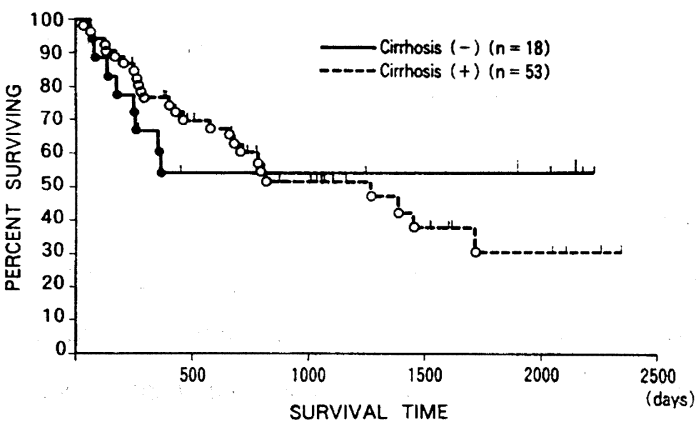

Fig. 10 Cumulative survival rates of resected cases according to coexistent hepatic cirrhosis.

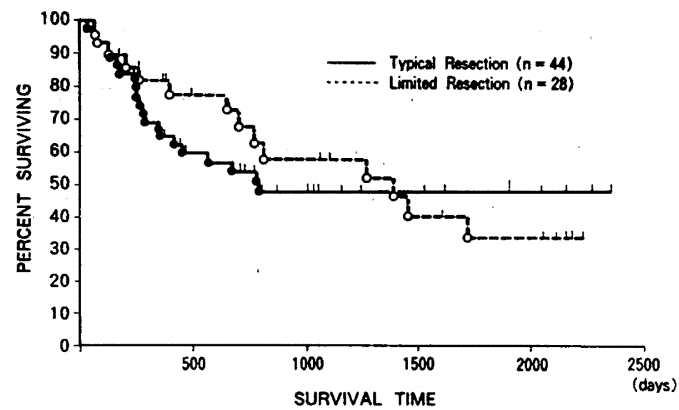

Fig. 12 Cumulative survival rates of resected cases according to resection type.

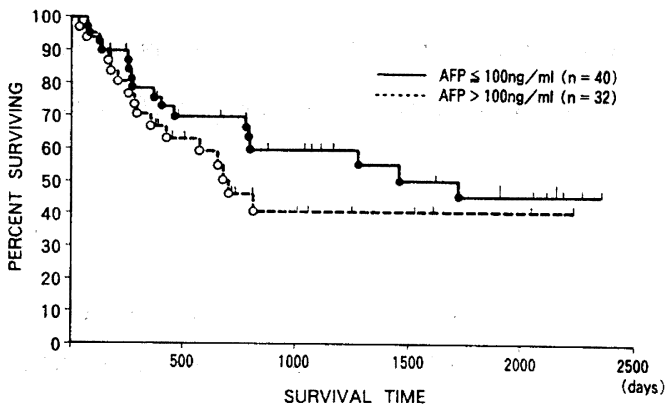

Fig. 9 Cumulative survival rates of resected cases according to serum AFP levels before the operation.

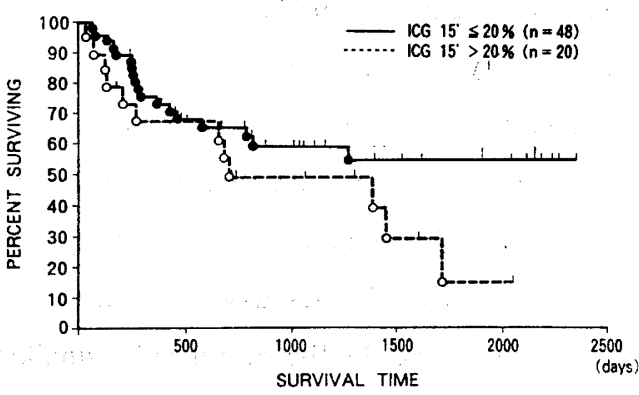

Fig. 11 Cumulative survival rates of resected cases according to preoperative ICG clearance.

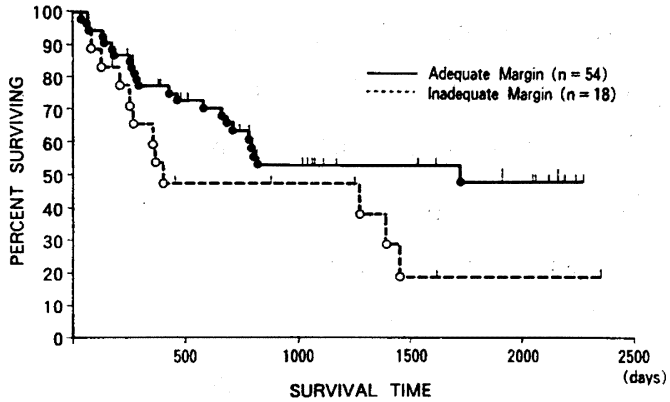

Fig. 13 Cumulative survival rates of resected cases according to tumor margin of resected specimen. 
observed for resection type or adequate/inadequate specimen margins (Fig. 12 and 13) although an adequate margin was associated with a better long-term survival rate as assessed by the Kaplan-Meier method $(P<0.05)$.

\section{Discussion}

In 1981 Fortner et al. ${ }^{6)}$ documented that the survival rates of patients undergoing hepatic resection for primary liver cancer were $85 \%$ at 1 year, $50 \%$ at 3 years, and $37 \%$ at 5 years. Similar results have been reported in the United States ${ }^{1,11)}$. In contrast, long-term survival rates have tended to be low in reports concerning Japan ${ }^{13,31)}$ and other Asian Countries 16, 42). In these latter cases most patients have cirrhosis, while this lesion is less common in Western series ${ }^{4}$. The resection of primary liver cancer has proved to be the best way to achieve long-term survival $\left.{ }^{33}, 38-40,44\right)$, its introduction for treatment of HCC being associated with significant prolongation of life, as also evident from the present results. Increased resectability and decreased operative mortality are therefore major goals for improvement of prognosis in primary liver cancer overall.

Lee et al. ${ }^{18)}$ reported remarkably longer survival rates in patients with asymptomatic as compared with symptomatic HCC. They also noted that even in the patients with symptomatic HCC, the $40 \%$ of cases associated with a typical tumor gross appearance, demonstrated a better prognosis. In the group with smaller, asymptomatic HCC the respective figure was $59 \%$. These findings suggested that two kinds of HCC might be present : the first with a typical gross appearance from the beginning until the end of the disease course has a better prognosis; the second, irrespective of its initial appearance, develops an atypical morphology as its grows and carries a poor prognosis. Therefore, the biology of the neoplasm itself may be the main determinant of prognosis.

On the other hand, factors other than the biological characteristics of the tumor i.e. capsule status, cell differentiation, such as liver disease background i.e. $\mathrm{HBsAg}$, coexisting liver cirrhosis also influence prognosis ${ }^{10,15}$. The Liver Cancer Study Group of Japan ${ }^{40}$ has investigated prognostic factors including tumor size, tumor number, capsular formation, venous involvement, and so on in a large number of HCC cases throughout the country, the findings being generally in line with those reported here. With regard to tumor size, a diameter of less than $5 \mathrm{~cm}$ appeared to be an important factor in predicting a good prognosis. Patients with a single nodule were found to survive longer than those with multiple tumors. Capsule formation was also an item of predictive evidence, the absence of a tumor capsule suggesting a poor prognosis. In contrast to the present series, patients without capsular invasion survived significantly longer than those with capsular invasion. Tumor emboli in the 
portal and hepatic veins were also reliable indicators of a poor prognosis.

Since accumulating findings have clearly shown that prognosis is closely related to the tumor size $3,20,37,40,43,44)$ and therefore, possible resection of early primary liver cancer is the key to improvement in survival. Follow-up studies using imaging modalities of highrisk patients which detected small and early carcinomas $\left.{ }^{2}, 9,14,32\right)$ were reflected by substantial lengthening of long-term survival rates ${ }^{19,20,41)}$. The present results thus further indicate that early detection and curative resection is the only way to enhance the long-term survival rate of cirrhotic cases with HCC, although the number of patients and the period of follow-up were not sufficient to allow estimation of definitive survival figures ${ }^{29,31)}$. It is therefore of great interest that the introduction of mass screening in high-risk areas of China has resulted in a remarkable increase in the numbers of patients detected with small and subclinical primary or recurrent tumors. Marked improvements in 5-year survival rates for HCC cases were clearly correlated to increased proportions of small lesions ${ }^{36,37)}$.

Since no significant differences in operative mortality rates exist between Asian and Western series, the rapid decline in long-term survival of the typical Asian patient suggests that HCC may be multicentric in cirrhotic livers. One of the problems in high risk areas for HCC have been the relatively high recurrent rate even after resection ${ }^{36)}$. Regular assessment of serum AFP levels and US examinations therefore remain important for patients undergoing HCC resection as well as for populations with a history of hepatitis and positive serum HBsAg.

Resection for subclinical recurrence of hepatic or pulmonary metastases had also proved to be effective in prolonging survival $28,36,45)$. Transarterial embolization or ultrasound-guided intralesional injection of ethanol was claimed to be the modality of choice for the treatment of nonresectable small HCC.

In conclusion, diagnosis and resection of small $\mathrm{HCC}$, primary or recurrent has been proved to be the best approach to achieving long-term survival. The resection rate might be higher, the operative mortality might be lower, and the 5 -year survival might be better. However, problems of coexisting liver cirrhosis particularly with non-compensated deterioration in liver function remain to be solved in the future.

\section{Acknowledgment}

The authors thank the members of Liver Cancer Study Group and Institute for Liver Research, Kansai Medical University, especially Drs. C. Koreeda and S. Uchiyama (Third Department of Internal Medicine) and Drs. S. K. Ha-Kawa and K. Harima (Department of Radiology) that supplied information and suggestion. 


\section{References}

1) Adson, M. A. and Weiland, L. H. : Resection of primary solid hepatic tumors. Am. J, Surg., 141, 18 20, 1981.

2 ) Chen, D. S., Sheu, J, C. , Sung, J. L., Lai, M.Y., Lee, C. S., Su, C. T., Tsang, Y. M., How, S. W., Wang, T.H., Yu, J.Y., Yang, T.H., Wang, C.Y. and Hsu, C.Y. : Small hepatocellular carcinoma ; A clinicopathological study in thirteen patients. Gastroenterol., 83, 1109 1119, 1982.

3) Chen, M. F., Hwang, T. L., Benjamin Jeng, L. B., Jan, Y.Y., Wang, C.S. and Chou, F. F. : Hepatic resection in 120 patients with hepatocellular carcinoma. Arch. Surg., 124, 1025 1028, 1989.

4) Chlebowski, R. T., Tong, M., Weissman, J., Block, J. B., Ramming, K. P., Weiner, J. M., Bateman, J.R. and Chlebowski, J.S. : Hepatocellular carcinoma : Diagnostic and prognostic features in North American patients. Cancer, 53, 2701 2706, 1984.

5 ) Ebara, M., Ohto, M., Shinagawa, T., Sugiura, N., Kimura, K., Matsutani, S., Morita, M., Saisho, H., Tsuchiya, Y. and Okuda, K. : Natural history of minute hepatocellular carcinoma smaller than three centimeters complicating cirrhosis ; A study in 22 patients. Gastroenterol., 90, 289 $\sim 298,1986$.

6) Fortner, J.G., Maclean, B. J., Kim, D.K., Howland, W. S., Turnbull, A. D., Goldiner, P., Carlon, G. and Beattie, E. J. : The seventies evolution in liver surgery for cancer., Cancer, 47, 2162 2166, 1981.

7) Franco, D., Smadja, C., Meakins, J.L., Wu, A., Berthoux, L. and Grange, D. : Improved early results of elective hepatic resection for liver tumors; One hundred consecutive hepatectomies in cirrhotic and noncirrhotic patients. Arch. Surg., 124, 1033 1037, 1989.

8. Gehan, E. : A generalized Wilcoxon test for comparing arbitrarily singly-censored samples. Biometrika, 52, 203 223, 1965.

9) Heyward, W. L., Lanier, A.P., McMahon, B. J., Fitzgerald, M. A., Kilkenny, S. and Paprocki, T. R. : Early detection of primary hepatocellular carcinoma; Screening for primary hepatocellular carcinoma among persons infected with hepatitis B virus. JAMA, 254, $3052 \sim 3054,1985$.

10) Hsu, H. C., Sheu, J.C., Lin, Y.H., Chen, D. S., Lee, C. S., Hwang, L. Y. and Beasley, R. P. : Prognostic histological features of resected small hepatocellular carcinoma (HCC) in Taiwan; A comparison with resected large HCC. Cancer, 56, 672 680, 1985.

11) Iwatsuki, S., Shaw, B.W. and Starzl, T. E. : Experience with 150 liver resections. Ann. Surg., 197, 247 253, 1983.

12) Kaplan, E. L. and Meier, P. : Nonparametric estimation from incomplete observation. J. Am. Stat. Assoc., 53, 451 481, 1958.

13) Kanematsu, T., Takenaka, K., Matsumata, T., Furuta, T., Sugimachi, K. and Inokuchi, K. : Limited hepatic resection effective for selected cirrhotic patients with primary liver cancer. Ann. Surg., 199, 51 56, 1984.

14) Kanematsu, T., Sonoda, T., Takenaka, K., Matsumata, T., Sugimachi, K. and Inokuchi, K. : The value of ultrasound in the diagnosis and treatment of small hepatocellular carcinoma. Br. J. Surg., 72, 23〜25, 1985.

15) Kishi, K., Shikata, T., Hirohashi, S., Hasegawa, H., Yamazaki, S. and Makuuchi, M. : Hepatocellular carcinoma ; A clinical and pathologic analysis of 57 hepatectomy cases. Cancer, 51, 542 $\sim 548,1983$.

16) Lee, N. W. , Wong, J. and Ong, G. B. : The surgical management of primary carcinoma of the liver. World J. Surg., 6, 66〜75, 1982. 
17) Lee, C. S., Chao, C.C. and Lin, T.Y. : Partial hepatectomy on cirrhotic liver with a right lateral tumor, Surgery, 98, $942 \sim 948,1985$.

18) Lee, C. S., Sung, J. L., Hwang, L. Y., Sheu, J. C., Chen, D. S., Lin, T. Y. and Beasley, R.P. : Surgical treatment of 109 patients with symptomatic and asymptomatic hepatocellular carcinoma. Surgery, 99, 481 490, 1986.

19) Li, G.H., Li, J.Q. and Zhan, Y.Q. : Surgical treatment of small primary liver carcinoma. Chin. Med. J., 99, 827〜828, 1986.

20) Li, G. H. and Li, J. Q. : Subclinical primary liver carcinoma. J. Surg. Oncol., 42, 181 183, 1989.

21) Lin, T.Y. and Chen, C.C. : Metabolic function and regeneration of cirrhotic and non-cirrhotic liver after hepatic lobectomy in man. Ann. Surg., 162, 959 972, 1965.

22) Liver Cancer Study Group of Japan (Ed) : The general rules for the clinical and pathological study of primary liver cancer. Kanehara Shuppan Co., Tokyo, pp. 19 22, 1987.

23) MacDonald, R.A. : Primary carcinoma of the liver ; A clinicopathologic study of one hundred eight cases. Arch. Intern. Med. 99, 266 279, 1957.

24) Makuuchi, M., Hasegawa, H. and Yamazaki, S. : Ultrasonically guided subsegmentectomy. Surg. Gynecol. Obstet., 161, 346 350, 1985.

25) Moody, F.G., Rikkers, L.F. and Aldrete, J.S. : Estimation of the functional reserve of human liver. Ann. Surg., 180, 592 598, 1974.

26) Nagao, T., Inoue, S., Mizuta, T., Saito, H., Kawano, N. and Morioka, Y. : One hundred hepatic resections ; Indications and Operative results. Ann. Surg., 202, 42 49, 1985.

27) Nagasue, N., Yukaya, H., Ogawa, Y., Sasaki, Y., Chung, C.Y. and Niimi, K. : Hepatic resection in the treatment of hepatocellular carcinoma. Report of 60 cases. Br. J. Surg., 72, 292 295, 1985.

28) Nagasue, N., Yukaya, H., Ogawa, Y., Sasaki, Y., Chang, Y.C. and Niimi, K. : Second hepatic resection for recurrent hepatpcellular carcinoma. Br. J. Surg., 73, 434 438, 1986.

29) Ohnishi, K., Tanabe, Y., Ryu, M., Isono, K., Yamamoto, Y., Usui, S., Hiyama, Y., Goto, N., Iwama, S., Sugita, S., Nomura, F. and Okuda, K. : Prognosis of hepatocellular carcinoma smaller than $5 \mathrm{~cm}$ in relation to treatment : Study of 100 patients. Hepatology. 7, 1285 1290, 1987.

30) Okamoto, E., Kyo, A., Yamanaka, N., Tanaka, N. and Kuwata, K. : Prediction of the safe limits of hepatectomy by combined volumetric and functional measurements in patients with impaired hepatic function. Surgery, 95, 586 591, 1984.

31) Okamoto, E., Tanaka, N., Yamanaka, N. and Toyosaka, A. : Results of surgical treatments of primary hepatocellular carcinoma : Some aspects to improve long-term survival. World J. Surg., 8, $360 \sim 366,1984$.

32) Okuda, K. : Early recognition of hepatocellular carcinoma. Hepatology, 6, 729 738, 1986.

33) Okuda, K. and The Liver Cancer Study Group of Japan : Primary liver cancers in Japan. Cancer, 45, 2663 2669, 1980.

34) Ozawa, K., Ida, T., Yamada, T. and Honjo, I. : Significance of glucose tolerance as prognostic sign in hepatectomized patients. Am. J. Surg., 131, 541 546, 1976.

35) Tang, Z.Y. : A new concept on the natural course of hepatocellular carcinoma. Chin. Med. J. 94, 585 588, 1981.

36) Tang, Z.Y., Yu, Y.Q. and Zhou, X.D. : An important approach to prolonging survival further after radical resection of AFP positive hepatocellular carcinoma. J. Exp. Clin. Cancer Res., 3, 359 366, 1984.

37) Tang, Z.Y., Yu, Y.Q., Zhou, X. D., Ma, Z.C., Yang, R., Lu, J.Z., Lin, Z.Y. and Yang, 
B. H. : Surgery of small hepatocellular carcinoma ; Analysis of 144 cases. Cancer, 64, 536 541, 1989.

38) The Liver Cancer Study Group of Japan : Primary liver cancer in Japan. Cancer, 54, 1747 1755, 1984.

39) The Liver Cancer Study Group of J apan : Primary liver cancer in Japan ; Sixth report. Cancer, 60, $1400 \sim 1411,1987$.

40) The Liver Cancer Study Group of Japan : Primary liver cancer in Japan : Clinicopathologic features and results of surgical treatment. Ann. Surg., 211, 277 287, 1990.

41) Watanabe, A., Yamamoto, H., Ito, T. and Nagashima, H. : Diagnosis, treatment and prognosis of small hepatocellular carcinoma. Hepato-gastroenterol., 33, 52 55, 1986.

42) Wu, M.C., Chen, H., Zhang, X.H., Yao, X.P. and Yang, J.M. : Primary hepatic carcinoma resection over 18 years. Chin. Med. J., 93, 723 728, 1980.

43) Wu, M.C., Zhang, X.H., Chen, H., Yao, X.P., Xu, G.N., Wu, B.W. and Yang, J.M. : Hepatic resection for primary liver cancer ; Report of 400 cases. Chin. Med. J., 99, 175 180, 1986.

44) Yamanaka, N., Okamoto, E., Toyosaka, A., Mitsunobu, M., Fujihara, S., Kato, T., Fujimoto, J., Oriyama, T., Furukawa, K. and Kawamura, E. : Prognostic factors after hepatectomy for hepatocellular carcinomas; A univariate and multivariate analysis. Cancer, 65, 1104 1110, 1990.

45) Zhou, X. D., Tang, Z.Y., Yu, Y.Q., Ma, Z.C., Yang, B.H., Lu, J.Z. and Lin, Z.Y. : Hepatocellular carcinoma ; Some aspects to improve long-term survival. J. Surg. Oncol., 41, 256 262, 1989. 